\title{
NOTAS DE INVESTIGACIÓN SOBRE JUVENTUD, CULTURA Y EDUCACIÓN: EL RELATO DE LOS ACTORES
}

\author{
MARCEL THEZÁ* \\ JORGE CASTILLO ** \\ EDUARDO CANDIA *** \\ ALAIN CARRIER $^{* *}$
}

\begin{abstract}
RESUMEN
El presente trabajo propone una revisión de un conjunto de fenómenos analizados en el marco del estudio «Juventud, cultura y educación» del Ministerio de Educación de Chile y de la Fundación Sistema de España. Concentrándose en la fase cualitativa de dicho estudio, se describen los aspectos más relevantes del relato juvenil en torno a las áreas de: a) caracterización y valores de los jóvenes; b) participación y ciudadanía y c) tendencias educativas. De igual forma, este trabajo finaliza sugiriendo una agenda tematizada de investigación que pueda profundizar ciertas dimensiones que este estudio no logró abordar a cabalidad, dada las restricciones propias de su naturaleza y propósito.

PALABRAS CLAVE: JÓVENES, EDUCACIÓN, CULTURA, VALORES, PARTICIPACIÓN

* Investigador del Centro de Investigación en Sociedad y Políticas Públicas, Universidad de los Lagos. E-Mail: marcel.theza@ulagos.cl.

** Académico de la Facultad de Ciencias Sociales e Historia de la Universidad Diego Portales, Santiago y consultor PNUD.

E-Mail: jorge.castillo@undp.org.

*** Profesional del Centro de Estudios, División de Planificación, del Ministerio de Educación de Chile. E-Mail: eduardo.candia@ mineduc.cl.

**** Investigador del Centro de Investigación en Sociedad y Políticas Públicas, Universidad de los Lagos. E-Mail: alain.carrier@ulagos.cl.
\end{abstract}




\title{
NOTAS DE PESQUISA SOBRE JUVENTUDE, CULTURA E EDUCAÇÃO: O RELATO DOS ATORES
}

\begin{abstract}
RESUMO
Este artigo propõe uma revisão de um conjunto de fenômenos analisados no âmbito do estudo «Juventude, cultura e educação», do Ministério da Educação do Chile e da Fundação Sistema da Espanha. Concentrando-se na fase qualitativa do referido estudo, são descritos os aspectos mais relevantes do relato juvenil sobre: a) caracterização e valores da juventude, b) participação e cidadania, e, c) as tendências educacionais. Da mesma forma, este trabalho sugere uma agenda tematizada de pesquisa que possa explorar certas dimensões que este estudo não conseguiu aprofundar, considerando as restrições próprias de sua natureza e objetivo.
\end{abstract}

PALAVRAS CHAVE: JOVENS, EDUCAÇÃO, CULTURA, VALORES, PARTICIPAÇãO

\section{RESEARCH NOTES ON YOUTH, CULTURE AND EDUCATION: THE STORY OF THE ACTORS}

\begin{abstract}
This paper proposes a revision of a set of phenomena in the framework of the study «Youth, culture and education» of the Ministry of Education of Chile and the Foundation System of Spain. By focusing on the qualitative phase of this study, we describe the most relevant aspects of the youth story around the areas of: a) characterization and values of youth, b) participation and citizenship, and c) educational trends. Similarly, this paper ends by suggesting a themed agenda of research facilities that it may deepen certain dimensions that this study did not address fully, given constraints of its nature and purpose.
\end{abstract}

KEY WORDS: YOUNG, EDUCATION, CULTURE, VALUES, PARTICIPATION 


\section{INTRODUCCIÓN}

EN EL PRESENTE ARTÍCULO se exponen los elementos más relevantes del análisis efectuado a tres grupos focales (focus group) realizados en el marco del estudio «Juventud, cultura y educación» ${ }^{1}$ del Ministerio de Educación de Chile, el Programa de Naciones Unidas para el Desarrollo (PNUD) y la Fundación Sistema de España.

Estos grupos focales fueron desarrollados con jóvenes de educación secundaria y la agrupación de los participantes fue intencionada de acuerdo a la pertenencia a tipos de establecimiento escolar; a saber: a) colegios privados sin aporte del Estado; b) colegios particulares subvencionados por el Estado; y c) colegios públicos dependientes del sistema municipal. Cada uno de estos grupos estuvo constituido por ocho jóvenes y su realización se llevó a cabo en el mes de abril del año 2012 bajo la coordinación y supervisión del equipo de profesionales responsables del proyecto, con el apoyo logístico (convocatoria y moderación) de la consultora Kronos Chile. ${ }^{2}$

La pauta de discusión de los grupos fue estructurada en base a ocho ejes centrales: a) identidad juvenil, b) educación y cultura escolar, c) asociatividad, d) sociabilidad, e) apoyo del sistema escolar, f) uso de nuevas tecnologías, g) violencia y alteridad, y h) valores.

En el marco que hemos descrito, este artículo presenta un conjunto de reflexiones surgidas exclusivamente de la fase cualitativa del estudio en cuestión; las que para su tratamiento serán sintetizadas en cuatro secciones: i) Características y valores del mundo juvenil, ii) Participación y ciudadanía juvenil, iii) Tendencias educativas relevantes y iv) Conclusiones: agenda de investigación. De esta forma, se busca poner en relieve un conjunto de fenómenos generales y transversales que aparecen reiteradamente en el discurso de los jóvenes y cuyas características y contornos nos permiten una mejor comprensión de los aspectos particulares que motivaron la realización de este estu-

1 Investigación en curso de realización, cuyo propósito es analizar comparativamente - Chile y España - los ajustes entre el funcionamiento de los sistemas educativos y las aspiraciones y expectativas de las nuevas generaciones de jóvenes. Dicha investigación busca aportar a la comprensión de este fenómeno a partir de una metodología mixta: a) una de tipo cualitativo a través de entrevistas grupales, y b) una de tipo cuantitativa-prospectiva a través de la aplicación de un cuestionario Delphi a treinta y dos expertos en el caso chileno y veintiocho en el caso español.

2 Equipo que coincide con los autores del artículo. 
dio. Estos elementos también constituyen focos de interés para todos quienes observan con atención la realidad e imbricaciones entre los jóvenes y el sistema educativo; lo anterior ya sea desde la perspectiva puntual de la investigación académica, o bien, desde el enfoque más funcional de las políticas públicas.

\section{CARACTERIZACIÓN Y VALORES DEL MUNDO JUVENIL}

a) Identidad juvenil, ¿cómo se ven los jóvenes?

...hoy día nosotros tenemos que decidir, de nosotros depende si queremos seguir estudiando o no seguir estudiando, onda, decidir nuestro futuro, más que nada aspecto laboral o también como familia porque podemos seguir otro camino, por ejemplo los estudios o si no simplemente dedicarnos al carrete (Mujer, colegio municipal).

Como aspecto relevante en este ámbito, se debe destacar el hecho de que los jóvenes señalen, sin distinción de colegio de origen, que el proyecto de continuación de estudios superiores es el «proyecto estructurante de sus vidas»; lo anterior tanto a corto como a mediano plazo. Otro tipo proyectos se supeditan más bien a la expectativa compartida de lograr una formación profesional, especialmente de tipo universitaria.

De igual forma, la concepción de juventud como una moratoria - donde se acumulan útiles sociales preparatorios para la vida adulta - es un elemento compartido por todos los jóvenes. Así, la juventud aparece como una etapa para prepararse y para tomar decisiones que tendrán efectos definitivos en la vida futura. Es en la juventud donde se van prefigurando las oportunidades y es preciso no dejarlas pasar. La juventud es una etapa de mayor libertad que permite tomar decisiones también con mayor autonomía. Sin embargo, esta libertad pareciese tener mayor potencialidad en unos que en otros. Los jóvenes de colegios privados no manifiestan un temor en cuanto al ejercicio de esta libertad y en cuanto a sus efectos; para ellos pareciese ser posible el error. En el caso de los jóvenes de colegios municipales y subvencionados, la libertad - principio altamente valorado- detona más bien una cierta angustia de no equivocarse al momento de decidir habiendo, por lo tanto, un sentimiento de tener menos derecho, menos margen, a tomar «malas decisiones». 
Es muy posible que los jóvenes de colegios municipales y subvencionados estén respondiendo afirmativamente a un llamado cultural a la autorrealización (Bajoit, 2003), en virtud del cual deben autónomamente tomar decisiones que a priori saben tendrán efectos definitivos en sus vidas. En este acto de tomar decisiones se materializa un ejercicio de la libertad que ellos, como lo hemos afirmado, no dejan de valorar. Sin embargo, los jóvenes comprenden que esta libertad no se da en términos absolutos y que el futuro no es sólo un futuro de oportunidades, sino también de obstáculos y dificultades. De la forma cómo ellos enfrenten estas «restricciones» y «resistencias» del Sistema, dependerán las trayectorias que logren construir. Los jóvenes de estos colegios se muestran muy conscientes de este proceso, y por ello este sentimiento de gran responsabilidad con las decisiones que deben ir adoptando.

No es menos relevante el señalar que para los jóvenes, o para sus familias, es un sacrificio de envergadura el continuar los estudios terciarios, por lo que acertar en esta decisión es clave. Constantemente se hace referencia al riesgo de terminar trabajando en algo que no sea satisfactorio y que no justifique el esfuerzo económico realizado (alto endeudamiento). La idea de «lo vocacional», como la posibilidad de pensar y de decidir sobre lo que quiere hacer y ser en el futuro, se encuentra cada vez más asentado en este segmento de la población, pero obliga - lo reiteramos - a una decisión con escaso margen de error.

En este misma línea resalta la manera en que los jóvenes tematizan la continuación de estudios terciarios. Para aquellos de colegios privados las acciones estratégicas son «ir a la universidad» $\mathrm{y}$ «estudiar una carrera», en cambio para los estudiantes de colegios públicos esta situación es tematizada como «sacar la carrera». La distinción es evidente: para los primeros es algo que se percibe como algo corriente y que sigue el curso normal de la vida; para los últimos esto aparece como algo que requiere de un esfuerzo mayor, a saber, desplegar una estrategia para poder tener la posibilidad de llevar a buen puerto aquello que se ha proyectado. La idea de «proyecto», como algo concientemente definido, está presente en todos los segmentos, pero es más demandante y estructurante de la identidad juvenil en los estudiantes de educación pública; posiblemente por la complejidad y dificultad de su desarrollo.

Este sentimiento de mayor presión no desdibuja el hecho de que esta juventud sea una juventud profundamente optimista en cuanto a 
su futuro individual; esto como una característica ya asentada y generalizada.

Otro aspecto interesante es la reiteración que hacen los jóvenes de colegios municipales y subvencionados del apelativo «crítico» como una característica esencial del período juvenil. Es en esta etapa donde, a juicio de ellos, se forma la facultad de observar la sociedad a través de este prisma de actitud.

En los estudiantes de educación privada los elementos que más se destacan se orientan más bien en torno a la idea de libertad: autonomía para actuar, valoración del emprendimiento y capacidad de articular proyectos variados.

Un último elemento que se menciona transversalmente como aspecto identitario de esta juventud, se relaciona con el rasgo de apertura a lo diverso; es decir, con la predisposición a aceptar y, en algunos casos más que en otros, a convivir con lo diverso, (homosexualidad, heterogeneidad de posturas ideológicas, etcétera). Lo anterior contrasta con la imagen que ellos tienen de la generación de sus padres cuando éstos fueron jóvenes, donde el grado de apertura, según su relato, era significativamente menor.

Como podemos apreciar, la autorepresentación que hacen los jóvenes de sí mismos parece dar cuenta de cambios mayores acontecidos en la sociedad en las últimas décadas. La relevancia atribuida a la capacidad personal para lograr los objetivos establecidos (autorrealización) es una característica epocal que caracteriza a las nuevas generaciones y que ideológicamente la propia sociedad ha reforzado con el propósito de mantener el status quo y atribuir a cada sujeto los resultados de su propia agencia. La sensación de poder lograrlo todo y que esto sólo depende del esfuerzo de cada uno, es una perspectiva funcional a un proyecto cultural que se ve potenciado con la promesa de la meritocracia. Ambos mensajes de la sociedad han calado hondo en la juventud actual, lo que se percibe ya que sus discursos identitarios se estructuran fuertemente desde esta perspectiva. Lo anterior se manifiesta más explícitamente al observar el valor que los jóvenes le otorgan a la educación. La educación se transforma en la herramienta principal sobre la cual se sientan las bases de los proyectos futuros; la educación permite autorrealizarse y sustentar - como lo hemos afirmado - una noción meritocrática de la sociedad. La identidad juvenil se define más nítidamente en tanto una etapa de preparación para la vida adulta, donde la educación es lo más relevante; es por ello que la condición de estudiante los define socialmente. 
En suma, la masificación de la educación y la cultura de la autorrealización son cambios societales que han redefinido radicalmente la condición juvenil. Si bien la figura de estudiante, como rol de preparación para autorrealizarse es significativa para los jóvenes, también emergen otras figuras identitarias. Así, el consumo como referente simbólico de la autonomía y la tolerancia a lo diverso como elemento propio de la cultura actual también se relevan como elementos que circunscriben la identidad juvenil actual.

Como lo hemos visto, la educación en general - y muy particularmente la educación superior - constituye para el conjunto de los jóvenes, independiente del establecimiento donde se estén formando, el instrumento fundamental para satisfacer las expectativas personales de desarrollo individual y de integración social. Si bien en ciertos jóvenes, fundamentalmente provenientes de los establecimientos privados, la idea de emprendimiento no necesariamente ligado a la formación profesional regular tiene una alta valoración, se tiende más bien a argumentar que la educación superior es «el camino que se debe transitar», siendo, a la vez, «el camino más fácil» si el objetivo es el éxito profesional a futuro. De esta forma, la expectativa por la formación de tipo superior es una expectativa compartida por todos los jóvenes, sin excepciones.

Sumemos a lo anterior el hecho de que los estudios en juventud realizados en Chile, particularmente la Encuesta Nacional de Juventud, ${ }^{3}$ vienen mostrando desde hace varios años la estabilización y consolidación de un fenómeno de «optimismo proyectivo» que se expresa muy nítidamente cuando a los jóvenes se les pide imaginarse a sí mismos en el escenario de un futuro próximo. En general, los jóvenes se imaginan mejor que como se encuentran en la actualidad, describiéndose a sí mismos inclusive mejor que el propio país (hay mayor optimismo individual que institucional). Este fenómeno se ratifica plenamente en los planteamientos realizados por los jóvenes participantes de los grupos focales. Ellos se ven de manera muy positiva a futuro, a pesar de que distinguen con claridad los problemas a los cuales tendrán que enfrentarse o verse expuestos.

\section{b) Metas y obstáculos}

Para los estudiantes de educación privada la meta es el despliegue de la autonomía y su «base de referencia» es lograr lo ya conquistado por

3 www.injuv.gob.cl/injuv2010/encuestas_juventud. 
sus familias. Los obstáculos fundamentales son, por una parte, el contexto de competencia que los enfrenta con otros proyectos, y, por otra parte, el riesgo de perder el rumbo: el desviarse del propósito trazado (donde «el carrete» aparece como potencial factor). En ellos, no hay una referencia explícita a elementos estructurales de la sociedad como grandes obstáculos.

Para los estudiantes de colegios subvencionados se insiste en la meta de formar una familia, aspecto ni siquiera mencionado por los jóvenes de colegios privados. Los jóvenes de colegios subvencionados al hacer referencia a los obstáculos para sus proyectos, manifiestan su temor frente a un mercado laboral en el cual se sienten en situación de desventaja, ya sea por su condición de jóvenes o simplemente por su poca experiencia laboral. Para los jóvenes provenientes de la educación municipal, la formación de una familia es visiblemente una meta. En ellos el discurso sobre los obstáculos adquiere mayor densidad al describir algunos escollos propios de la condición juvenil (v.g.: extrema dependencia de los padres, asimetría de los derechos) y también ciertas restricciones estructurales como lo es muy concretamente la situación de desempleo juvenil.

Los jóvenes en general se refieren al desarrollo laboral y al cumplimiento de la «vocación» como metas esenciales. Así se define al trabajo como una labor que debe ser elegida concientemente («vocación»), donde se debe ser competente para poder llevar a una buena vida; es decir que debe ser retribuida con una remuneración que permita un buen pasar.

También aparece con fuerza, como meta o indicador de éxito, el empezar a adquirir ciertos bienes (una casa, un auto, etcétera), los que funcionarían como externalidades simbólicas de la autonomía deseada; autonomía que además permite generar las condiciones necesarias para construir una familia. Esta imagen está más presente en estudiantes de colegios públicos y subvencionados.

La idea de «perder el rumbo», como lo hemos descrito en relación a los jóvenes de colegios privados, aparece con distinta intensidad también en los otros jóvenes; en este caso se menciona como obstáculo el perderse en el exceso, en el «carrete».

Es interesante constatar que los principales obstáculos mencionados - sobre todo por los estudiantes de colegios públicos y subvencionados - tienen que ver con aquello que impide o dificulta lograr terminar la educación terciaria. La escasez de recursos económicos o el no ser capaz de obtener el título profesional deseado por mal desempeño 
académico son los principales temores sobre este punto. En este sentido, se cree que logrando la obtención de dicho título, casi automáticamente (sin mediar otras instancias) se desencadenan una serie de situaciones positivas socialmente hablando (establecer una familia, adquirir autonomía, obtener un buen trabajo, ser valorado socialmente, etcétera), lo que plantea una alta expectativa sobre los efectos del proceso de formación profesional. Lo anterior da cuenta de lo enraizada que se encuentra en la población juvenil la ideología de la meritocracia, donde se cree que por tener un buen desempeño educativo (traducido en acceso a la educación superior) se va a acceder a una posición más alta en la estructura social. Los jóvenes estiman que el acceso a la universidad generará ese paso prácticamente de manera automática. Todo ese imaginario tiene su base en lo que provocaba la educación superior hace veinte o más años atrás, sobretodo para «los becarios» o «pobres meritorios», los que debido a su desempeño y el ingreso logrado a la educación superior conseguían efectivamente movilidad social (Dubet, 2005). Hoy la educación terciaria se ha masificado, tanto respecto a la población beneficiada como respecto a la cantidad y variedad de la oferta institucional existente (no todas efectivamente movilizantes), lo que acarrea consecuencias evidentes como la pérdida de valor del diploma - más aún en aquellas generadas en instituciones de menor valoración social - e incertidumbre respecto a la movilidad social. Estudios recientes muestran que hoy en Chile esta masificación en la educación superior puede ser claramente observada considerando a aquellos que son los primeros en sus familias en acceder a este tipo de educación. Así se menciona que un sesenta y cuatro coma cuatro por ciento $(64,4 \%)$ de los estudiantes en educación terciaria son primera generación (Castillo y Cabezas, 2010).

\section{c) Referentes significantes para los jóvenes}

...en mi caso, que puede ser como la PSU, obviamente al primero que voy a acudir es a mi papá más que nada esa es la persona que va a estar conmigo en el momento de ayudarme a razonar lo que quiero (Hombre, colegio privado).

En cuanto al tema de la confianza, prácticamente todos los jóvenes dudan al momento de intentar identificar referentes que la ilustren. Finalmente los estudiantes de colegios privados se inclinan por la figura los «emprendedores anónimos de cada día» que se sobreponen 
a las dificultades. Para los de colegios municipales y subvencionados, la confianza está depositada en los referentes significantes de la familia o círculo cercano; es su ejemplo la persona que orienta y motiva. Planteado desde otra perspectiva, todo indica que los estudiantes de colegios privados tienen una mayor inclinación a definir un «ideal tipo» que sirve de aliciente (como lo hemos descrito, es el caso del «emprendedor», «el esforzado», «el perseverante», «el que sigue sus impulsos», etcétera). En los jóvenes de colegios municipales y subvencionados, este proceso es menos abstracto porque se produce a partir de «personas muy en concreto» que se ubican fundamental y preferentemente en el espacio que produce mayores niveles de confianza, a saber, la familia.

Como antes lo hemos afirmado, los modelos descritos por los estudiantes de establecimientos subvencionados y públicos refieren a ejemplos típicos de resiliencia y movilidad social. Principalmente ellos están relatados en términos de personas cercanas (generalmente algún familiar cercano en términos etarios, como un hermano) que estudian o lograron terminar la educación superior y que trabajan, con lo cual comienzan a adquirir lo primeros bienes-signos de autonomía. Generalmente, son modelos que encarnan el esfuerzo personal. Estos últimos referentes son de alguna forma modelos que pareciesen reforzar una cierta idea de meritocracia, lo que hace plausible seguir creyendo en las oportunidades que entrega la sociedad. El mensaje es claro: «hay que esforzarse, ya que depende de uno poder aprovechar estas oportunidades». En este sentido es fundamental «sacar la carrera», requisito necesario para que esa posibilidad efectivamente se pueda desplegar.

Todos los jóvenes coinciden con que la familia ocupa un lugar de privilegio como referente al momento de tomar una decisión. En este marco, todo indica que la familia es un factor de seguridad que compensa la eventual precarización de las redes sociales y asociativas. Frente a la dificultad para crear vínculos sociales, los jóvenes tienden a replegarse hacia los vínculos familiares. Otros planteamientos ya han mostrado de que en rigor los jóvenes se repliegan hacia la familia en cierto sentido para «escapar» a la sociedad donde «la familia es un refugio» (Lechner, 2004). Mientras que entre los jóvenes pertenecientes a colegios privados, otros referentes al momento de tomar «decisiones importantes» son los profesores y los amigos. En el caso de los jóvenes de educación subvencionada y municipal este segundo referente sólo se concentra en el grupo de amigos. 
En todo caso, decisiones relativas a temas de estudio, las que son consideradas como las decisiones más significativas, son habitualmente tomadas junto a la familia. Por el contrario, las decisiones relativas a la esfera afectiva y de la sexualidad son normalmente tratadas junto al grupo de pares (amigos, compañeros de colegio, etcétera).

\section{d) Estructuración de redes de sociabilidad}

A través de sus testimonios los jóvenes muestran tener una sociabilidad muy limitada y fragmentada. En el caso de los estudiantes de colegios privados, sus redes están estructuradas en base a la familia extendida (lo anterior muy nítidamente), el colegio y el barrio. En el caso de los jóvenes de colegios subvencionados y municipales, las dos fuentes de creación de redes son bien precisas: el colegio y el barrio.

Si sostenemos que la sociabilidad es en efecto un principio de apertura y de trascendencia social que permite conocer y asimilar lo que es por su propia naturaleza desconocido, y si afirmamos también que lo propio de la sociabilidad es la capacidad de fundar vínculos que vayan más allá del contexto identitario directo, estos jóvenes aparecen muy afianzados en una especie de comunitarismo territorial y social que no les permite claramente resolver los problemas de la diferencia y la alteridad a través de redes más amplias. En efecto, se aprecia que estas redes son puestas a disposición fundamentalmente de actividades de esparcimiento (conversar, ir al mall, juntarse en la plaza, ir en grupo al cine y fundamentalmente carretear). Un aspecto interesante es que para efectos del carrete los estudiantes de colegios subvencionados se refieren insistentemente al «carrete en la plaza»; es decir, hay un uso más frecuente de este tipo de espacio público y al riesgo que esto conlleva. En el caso de los jóvenes de colegios privados la situación es distinta; la sociabilidad se construye en un contexto aparentemente más privado.

Las redes sociales participan de este proceso, pero sólo permitiendo una mayor comunicación entre actores de redes ya formadas. No hay referencias a procesos de exploración, de búsqueda, de otras redes, sino más bien a la consolidación de redes ya predefinidas.

Nuevamente el discurso de los jóvenes los muestra muy determinados simbólicamente por el espacio familiar. Este es el espacio protector, el redil que eventualmente hace menos angustioso el proceso de contacto con nuevos entornos que son por su naturaleza desconocidos (Guell, 2008). 
Por lo mismo, los vínculos que se crean con los amigos del colegio y del barrio están muy impregnados por la necesidad de «reconocerse» en una misma experiencia, en un mismo sistema de valores o en una misma visión de la sociedad. La sociabilidad, en este caso, se acerca más a la construcción de un «pequeño mundo» que se pueda administrar sin verse obligados a esfuerzos excepcionales de apertura o de trascendencia social.

\section{e) Alteridad y violencia}

...es que nosotras las mujeres somos más de frente, somos más «oye que te pasa tal por cual»; entonces nosotros vamos directo al choque, en cambio los hombres dicen «oh este me cae mal» y dice, pero «hola como estás» y por detrás «oh este loco me cae súper mal»; así son los hombres (Mujer, colegio subvencionado).

Violencia social: características y causas. Todos los jóvenes coinciden con el hecho de que la sociedad chilena es una sociedad donde las experiencias de violencia se multiplican. Hay, por lo tanto, violencia como modalidad de relación y ella tiene efectos permanentes en la forma como los chilenos construimos nuestros vínculos (Thezá y Reinoso, 2005). No obstante este marco general, hay ciertas distinciones al momento de intentar describir las causas que dan origen a la violencia.

Para los jóvenes provenientes de colegios privados las causas son claras: la falta de educación, la debilidad del entorno familiar y las dificultades para integrarse a la sociedad, para sentirse parte de ella. Los jóvenes de colegios subvencionados ponen el acento en una visión más esencialista, planteando que la naturaleza de las personas es la violenta; por lo tanto es un hecho que reviste de una cierta «normalidad». Son los jóvenes provenientes de colegios municipales quienes aluden a que la violencia sería una respuesta frente a la intolerancia y al malestar social que las personas viven día a día.

Violencia en el colegio. Todos los jóvenes coinciden en cuanto a que la violencia está permanentemente presente en el colegio. Los estudiantes de colegios privados y municipalizados ponen un acento preferente en la violencia psicológica y el bulling, siendo la violencia física un elemento mayormente planteado por los estudiantes de colegios subvencionados. Son los propios jóvenes de colegios subvencionados quienes hacen referencia a un fenómeno que les sorprende y les 
molesta; a saber, el aumento de situaciones de violencia física entre mujeres. Los estudiantes de colegios particulares hacen la salvedad de que en sus establecimientos la situación está controlada y hay una tendencia a la disminución.

En relación a los fenómenos de violencia entre profesores y alumnos (en ambos sentidos), todos los jóvenes, independiente de su colegio de origen, plantean que esta es una realidad que existe en diversas intensidades; sin embargo, ellos también son unánimes al señalar que esto no es normal y que las relaciones entre alumnos y profesores dibieran siempre estar caracterizadas por una lógica de respeto en ambas direcciones.

Los ejemplos de violencia que aquí se refieren están relacionados con formas de abuso de la autoridad por parte de los profesores como recurso de poder para mantener el orden, pero también como una eventual manifestación de frustración del profesorado cuando el proceso educativo experimenta dificultades.

Un aspecto interesante es la función de contención frente a eventuales actos de violencia entre profesores y alumnos. Según los jóvenes de los colegios privados, son los padres los que rápidamente actúan («mis padres no lo aceptarían»), siendo, en el caso de los colegios particulares y subvencionados, responsabilidad de los propios jóvenes el hacerse respetar.

Experiencias de violencia. Las experiencias personales de violencia vividas de manera más significativa por los jóvenes participantes de los grupos focales son los acontecimientos de violencia en el «carrete» y en las actividades festivas en el caso de los jóvenes provenientes de colegios privados; el hostigamiento psicológico y las «tallas» (bromas) en el caso de la educación subvencionada; y el abuso de autoridad en el caso de los jóvenes de colegios municipalizados.

Habitualmente el tema de la violencia es abordado como si él fuese una mera perturbación del orden social que debe ser enfrentado a través de mecanismos acertados de control. Esta concepción del trastorno social tiende normalmente a dividir a la sociedad entre sanos y enfermos, entre buenos y malos. Sin embargo, el discurso de los jóvenes se orienta más bien hacia una concepción de la violencia que la instala en las formas de relación social.

Aquí la violencia aparece como la expresión de conflictos, pero también de intereses que son antagónicos en el plano psicosocial, político y/o cultural. Por lo tanto, la violencia se transforma en el resulta- 
do de la incapacidad de enfrentar situaciones de conflicto — una idea distinta, la disputa por un/a pololo/a, una participación en un grupo distinto, etcétera- a través de mecanismos legítimos como la palabra, los ritos, las mediaciones simbólicas, etcétera.

La violencia no es en este caso un atributo de lo juvenil y los discursos de los jóvenes lo reafirman. La violencia aparece más bien cuando hay incapacidad para desarrollar instrumentos de resolución de conflictos y/o satisfacción de las gratificaciones personales. En este caso muchas veces son los grupos de pares quienes terminan reforzando circuitos de riesgo que hacen difícil la implementación de lógicas no violentas. Esto muestra lo importante de concentrarse en el mejoramiento de los factores de cohesión que se fundan en la idea de «convivencia». En este marco no se debiera descuidar el hecho de que escuela, barrio, familia y sociedad tienen que ser abordados integralmente. De hecho, las acciones de mayor exposición a la violencia son, de acuerdo al discurso de los jóvenes, vividas fuera del colegio.

Los jóvenes viven el día a día en espacios bien precisos y, por lo tanto, sus conductas no son diferentes a las redes que dan sentido a sus prácticas. Fortalecer esas redes debiese permitir un diálogo más estrecho entre identidad y alteridad.

\section{f) Valores}

...porque tampoco va a plantear una discusión con ellos porque yo también tengo amigos homosexuales y súper buena onda y todo pero tampoco estoy de acuerdo con la homosexualidad por cosas de principios y todo, pero tampoco le hago la cruz porque sé que son personas y todo (Mujer, colegio municipal).

Para manifestarse frente a este tema, los jóvenes debatieron en torno a dos tópicos esenciales: la relación con jóvenes extranjeros en el aula y sus impresiones en relación a la homosexualidad.

Para todos los jóvenes opera una «tolerancia selectiva» que establece que la diferencia cultural es aceptada y reconocida sin problemas, pero el límite es que la realidad que se acepta no afecte y no interfiera demasiado la condición de quien la juzga. Por ejemplo, el tratamiento de «lo extranjero» obedece a este patrón puesto que él está revestido de un carácter exótico y atractivo que es muy funcional a esta búsqueda de referencias culturales más amplias que el propio país. Sin embargo también se expresa la idea de que «los acepto, pero que no vengan a criticar 
a mi país». Hay que precisar que las respuestas planteadas tienen un sesgo importante y es el hecho de que muchos de los jóvenes extranjeros provienen de algún tipo de programa de intercambio o son de nacionalidades distintas a las que frecuentemente se ven enfrentadas a la discriminación en nuestro país (países de frontera).

En cuanto a la homosexualidad se expresa el mismo criterio: «lo acepto, pero que no se metan conmigo». Los estudiantes de colegios privados son quienes manifiestan mayores reticencias para describir el fenómeno que se vive en sus colegios dado que señalan que en ellos se preserva el criterio de que la dimensión privada no se debe exponer públicamente. El discurso de los jóvenes los muestra con un espíritu de apertura, de comprensión y de tolerancia hacia la idea de diversidad. En la construcción de sus afirmaciones se mezclan eventualmente aspectos relativos a un contexto políticamente correcto que censuraría un discurso distinto. Sin embargo, la descripción de situaciones puntuales que ellos relatan indica que estas percepciones están bien asentadas y que ellas difieren significativamente de aquellas que tienen y expresan las generaciones anteriores, sus padres preferentemente.

De igual forma se aprecia que los juicios de los jóvenes están cada vez más desprovistos de restricciones moralizantes que regulaban muy significativamente en el pasado la opinión de las personas. Hoy los jóvenes se sienten más libres, más autónomos, para plantear opiniones personales y también opiniones situacionales sin que deba mediar un referente institucional preciso que los oriente, por ejemplo, familia, iglesia, etcétera.

No obstante lo anterior, la tolerancia tiene fronteras, tiene límites y estos límites efectivamente están definidos por una selectividad que podría explicarse bajo la siguiente fórmula: «acepto la diferencia mientras esa diferencia no me afecte, no influya en mi vida». En el discurso de los jóvenes de colegios privados es «acepto la homosexualidad mientras ésta se dé en el espacio privado y no en el espacio público de mi colegio», o en el discurso de los jóvenes de colegios municipales «acepto la homosexualidad, pero no quiero que me involucren en sus conductas». En base a los discursos de los estudiantes, da la impresión de mayor tolerancia en los estudiantes de establecimientos particulares subvencionados y sobre todo de establecimientos municipales. 


\section{PARTICIPACIÓN Y CIUdAdAnía JUVENIL}

a) Los jóvenes y los sistemas de representación: el problema de la confianza

Los jóvenes confirman el diagnóstico ya generalizado y compartido sobre la desconfianza hacia los actores político-institucionales en todos sus niveles. Hay desconfianza, pero hay ante todo desconocimiento del funcionamiento del sistema institucional y de sus representantes. En este campo no hay grandes distinciones entre jóvenes de un sistema escolar u otro, siendo sólo relevante el hecho de que los jóvenes de colegios municipales y subvencionados manifiestan mayor conocimiento e interés por las autoridades del nivel local (INJUV, 2011).

Precisemos que este estudio no tuvo como finalidad profundizar el análisis sobre la relación entre jóvenes y política y, por ello, se limita sólo a poner en evidencia el problema ambiental de la confianza hacia las instituciones del sistema democrático como contexto de comprensión de otro tipo de fenómenos más pertinentes con el espacio escolar. Como ya lo hemos afirmado, la confianza en las autoridades es muy débil. A lo anterior se suma el hecho de que paradojalmente los jóvenes tampoco conocen a las autoridades que debiesen ser depositarias formales de su confianza. Los jóvenes conocen al Presidente de la República y tienen juicios diversos sobre su comportamiento, pero a nivel local presentan dificultades para identificar a los alcaldes. Son los jóvenes de colegios subvencionados y municipales quienes muestran un mayor conocimiento de sus autoridades locales.

Los motivos por los cuales desconfían son variados, pero se concentran fundamentalmente en la percepción de que la política es una actividad donde prevalecen intereses propios de los actores políticos que no necesariamente interesan a los ciudadanos. La política tiene un rostro oscuro, secreto, inasible. La propia referencia a la competencia política es engañosa ya que el balance de los jóvenes es que, desde el punto de vista práctico, unos y otros son lo mismo; no hay una diferencia que se pueda apreciar claramente.

\section{b) Los jóvenes y el reproche a la sociedad}

Los jóvenes reprochan muchos aspectos que señalan como incómodos, molestos, desagradables, en la sociedad. Gran parte de esos reproches se concentran en el factor «competencia». Sin embargo, la 
óptica desde la cual se critica la competencia no es la misma para todos ellos. Para los jóvenes de los colegios privados, la extrema competencia es una amenaza que pone en tensión las trayectorias deseadas, fundamentalmente en el campo profesional: «competir con otros me quita posibilidades». En este contexto, la crítica es funcional; no está dirigida a la idea de competencia en sí, sino a las dificultades que presenta en el desarrollo personal. Por el contrario, en el caso de los jóvenes de colegios municipales y subvencionados, el reproche es de orden ético; a saber, la extrema competencia desnaturaliza el espacio escolar y destruye los vínculos de cooperación de la sociedad. En este caso, no es sólo que la competencia «no me convenga»; el tema de fondo es que «no me gusta». Respecto a este último aspecto, es posible reconocer una crítica al sistema y al rol de la educación en ese contexto. Este modelo es percibido como un modelo que obliga a actuar «anti natura». Que prepara en las habilidades y competencias necesarias para entrar en la «máquina» (sociedad y sus demandas), la que en palabra de los estudiantes «te envuelve y no te deja salir y disfrutar de la vida». Esta sería una máquina que te hace trabajar para sobrevivir (misma idea que el hecho de endeudarse para estudiar y así trabajar para poder pagar la deuda contraída). La educación desde esta perspectiva sería funcional a esa lógica que va en contra del desarrollo integral de las personas. Este es un discurso más asentado en los estudiantes de colegios municipales y en parte por aquellos de colegios particulares subvencionados.

\section{c) Participación: colegio y sociedad}

...sí, con un año y chao, no por el tema de las marchas ni esas cuestiones. Así si yo tengo que igual me movía por esa cosa, pero el resto del centro de alumnos era nadie más, no estaba preocupados de sus líos nomas, del aniversario del colegio y chao y nada más (Hombre, colegio subvencionado).

Los jóvenes manifiestan mayoritariamente tener poca participación en las estructuras asociativas naturales de sus colegios (centro de alumnos, consejos de estudiantes, etcétera), concentrando estas actividades más bien fuera del espacio escolar. En rigor, la participación desarrollada fuera del sistema escolar está fundamentalmente asociada al periodo vital de los jóvenes a través de actividades deportivas, religiosas, de voluntariado o de simple esparcimiento. 
Otro tipo de actividades que podríamos definir como «emergentes» (grupos organizados por una causa social, grupos altermundialistas, etcétera) no son mencionados por los jóvenes en ninguno de los casos.

Sin embargo, es interesante el hecho de que la referencia que los jóvenes de colegios privados hacen sobre la participación se remita a la idea de «adquisición de una mayor experiencia» que debe servir en la vida futura; todo esto bajo una perspectiva claramente instrumental y más individualista. En el caso de los jóvenes de colegios municipales y subvencionados se participa, por el contrario, para desarrollar cooperación y compañerismo; pero, lo más importante, se participa para ejercer «derechos». Este concepto surge con mucha nitidez en estos jóvenes en particular

Los a priori sobre los jóvenes en temas de participación a menudo nos llevan a pensar que ubicándose esta generación en el corazón del surgimiento de nuevos temas de interés ciudadano, como lo son objetivamente los derechos de las minorías sociales, los derechos medioambientales, etcétera, este nuevo cuadro de intereses sociales y culturales debiese traducirse en un aumento de la participación; fundamentalmente aquella que se desarrolla al margen de las instituciones de representación tradicionales. Sin embargo, la realidad parece ser otra, puesto que los jóvenes no plantean tener una participación asociativa permanente y activa. Por lo menos esto no es una característica central en ellos.

De igual forma, puesto que este estudio se ha contextualizado bajo la premisa de la existencia de cambios culturales generales que tienen un impacto objetivo sobre los jóvenes, también se podría esperar que esta dinámica de cambios se instale en el campo de la participación. Bien por el contrario sus iniciativas de participación son más bien tradicionales y ellas responden a necesidades muy precisas del periodo juvenil como elemento formador de identidad.

Sin embargo, no es prudente desatender otros elementos que se desprenden de los relatos juveniles y que son de una gran importancia; entre ellos destacaremos dos. El primero es la incorporación de la noción de «defensa de derechos» (no precisados) como un aliciente a la participación; lo anterior principalmente en el caso de los estudiantes de colegios no privados. Lo segundo es que la débil «participación asociativa» efectiva se da en un contexto de desconfianza institucional muy profunda, y por ello el sistema de representación no colabora en el proceso de producción de nuevos ciudadanos más activos, más involucrados y también más vigilantes. 
No obstante aquello, práctica y discurso juveniles son en muchos campos contradictorios y por ello pudiésemos pensar que hay aún un proceso en ajuste, en redefinición, que no nos permite hablar de modalidades de participación plenamente definidas y acabadas.

\section{TENDENCIAS EDUCATIVAS}

...no todos reciben la misma educación, o sea si uno se pone a comparar entre un colegio particular subvencionado y uno particular, la educación no es la misma. O sea, me refiero no sé cómo explicarlo pero el nivel académico que se entrega en cada colegio es muy distinto. Por ejemplo yo estoy en un colegio particular subvencionado y tengo que hacer un preuniversitario, pero en cambio en un colegio que sea privado no lo va a necesitar porque va a estar preparado. Entonces esa desigualdad no corresponde (Mujer, colegio subvencionado).

\section{a) Percepción de la calidad del sistema}

Un fenómeno ampliamente compartido por todos los jóvenes, independiente de su colegio de origen, es el juicio taxativo de que «la educación en general - la de los otros - es de mala calidad, pero la propia es buena». Esto ya ha sido puesto en evidencia reiteradamente y muestra la dificultad de los jóvenes para aplicar juicios objetivos cuando la realidad se presenta muy próxima. En rigor, los jóvenes escuchan cotidianamente que la educación chilena es de mala calidad, pero no poseen mayores instrumentales evaluativos que les permitan analizar la propia, $\mathrm{y}$, por lo tanto, su filtro de actitud pareciese ser más bien afectivo.

Los jóvenes no logran formular a qué equivale «la calidad» en la educación, menos aún cuáles serían sus indicadores. Sin embargo, de sus intervenciones se desprende que existe un criterio común: la educación - tanto básica como secundaria - debe preparar de manera muy instrumental al ingreso a la educación superior, y a la superación del hito que es la prueba de selección universitaria. En rigor, cuando la educación no es de buena calidad, los estudiantes se ven obligados a asistir a un preuniversitario que llene las lagunas producidas en el colegio. De esta forma el preuniversitario se transforma, prácticamente, en un nuevo nivel de educación formal propio del modelo chileno. Esta afirmación está presente de manera más intensa en los estudiantes de colegios subvencionados.

En el caso de los jóvenes de colegios privados, la calidad también está asociada a los conocimientos y competencias de los profesores y a 
las condiciones del contexto educativo (v.g., cantidad de alumnos por curso). En este sentido se configura la idea de calidad desde una noción más amplia, compleja y procesal que en las apreciaciones anteriores.

No obstante lo anterior, es posible hacer ciertas distinciones. Para los jóvenes de educación privada, la educación es mala puesto que la referencia es la realidad escolar de otros países que, en su juicio, tiene un mejor «rendimiento». Para los jóvenes de educación subvencionada la educación es mala, porque es desigual (no es la misma en calidad para todos). Para los jóvenes de educación municipal, la educación es mala, porque fomenta una insana competencia. En ambos casos, el reproche es nuevamente de naturaleza eminentemente ética; fenómeno que ya hemos destacado anteriormente.

Cercana a esta última perspectiva, pero en cierta forma contradictoria con la apreciación inicialmente descrita, emerge una apreciación transversal, pero más fuertemente encarnada en el discurso de los estudiantes de colegios públicos, que nos muestra que hoy estamos en presencia de una educación muy pragmáticamente dirigida a «lo cognitivo», a aquello que es más funcional al sistema económico reinante y que ha olvidado el carácter integral que debiese tener la educación. Esta crítica se evidencia en lo olvidadas que están algunas disciplinas, sobretodo aquellas más humanistas, en la oferta curricular actual: poco deporte, arte, educación cívica, etcétera. En concreto, se habla de un sistema impersonal que fabrica sujetos funcionales a la «máquina» (sociedad) como se mencionó anteriormente; una educación para el desarrollo económico y no para el desarrollo humano en palabras de Nussbaum (Nussbaum, 2010)

Es importante relevar el carácter aparentemente contradictorio de las opiniones de los estudiantes. Por un lado, piden una mejor educación que se enfoque en preparar eficazmente para la prueba de selección universitaria, pero, a la vez, critican este tipo de educación que no da más oportunidades para desarrollarse integralmente. En las palabras de los propios estudiantes es posible clarificar primariamente esta paradoja; si bien no aceptan la racionalidad que le impone el sistema por encontrarlo deshumanizante, reconocen que no tienen la fuerza necesaria para cambiar dicho modelo, razón por la cual exigen que la educación, con sus sesgos y todo, sea capaz de actuar equitativamente entregando la educación necesaria a todos para tener las mismas posibilidades de ingresar a la educación superior y de esa forma poder ser «competente» para el Sistema. 


\section{b) Pertenencia de contenidos}

Reinterpretando las opiniones de los jóvenes, podríamos señalar que, más allá del juicio personal sobre la pertinencia de los cursos que no son de su agrado o donde el rendimiento académico es menor, se abre una expectativa de aquello que debiese lograr un mayor desarrollo en el proceso formativo global. Para los jóvenes de colegios privados nos referimos a las habilidades sociales vinculadas a la capacidad de expresar ideas, al mejor uso de nuevas tecnologías, al deporte y al arte. En el caso de los jóvenes de colegios subvencionados es claramente el deporte y en el caso de los jóvenes de colegios municipales es la historia y los idiomas, preferentemente el inglés.

Otro aspecto importante es el relativo a la presencia de contenidos de sexualidad en el colegio. En torno a esto todos los jóvenes insistieron en la importancia de que este tema se aborde seriamente en el currículum, planteando su percepción en cuanto a que el elemento inhibidor del tratamiento de estas materias en el colegio no es la restricción institucional (neutralidad) o ideológica de los establecimientos, sino fundamentalmente el comportamiento de los propios padres, quienes constantemente están censurando la realización de estas actividades en el aula. Incluso, cuando estos temas son abordados curricularmente en biología, generalmente son anacrónicos ya que muchos de los estudiantes ya han iniciado su vida sexual

Respecto a otro elemento donde la discusión pudo ser profundizada - la educación cívica - , los jóvenes señalan no contar con una formación adecuada en este campo, limitándose sólo a algunas referencias vistas en clases de historia. Todos ellos consideran que la formación ciudadana es fundamental y que debiese ser trabajada más profundamente en sus colegios. Los estudiantes de educación privada plantean que la educación cívica debiese concentrarse en proporcionar información (quiénes son las autoridades, cómo funcionan las instituciones, etcétera), mientras que los estudiantes del nivel municipal insisten en la necesidad de que la educación cívica se concentre muy particularmente en difundir los derechos de las personas. Ambos grupos critican el no recibir más educación cívica; ambos grupos la demandan.

Como ya se mencionó anteriormente, estamos en presencia de una demanda paradojal: Se piden contenidos más prácticos y funcionales con el perfil de salida, pero a la vez se demanda mayor integralidad en la formación (más educación cívica, talleres extraprogramáticos, etcétera). 
Se percibe también una demanda incipiente por parte de los jóvenes de contar con una mayor autonomía en la configuración de sus mallas de estudios. Específicamente se pide más libre elección respecto a las asignaturas a tomar, dando la posibilidad de elegir asignaturas que tradicionalmente han sido concebidos como de distintas áreas del saber.

\section{c) Percepción hacia los profesores}

Todos los jóvenes manifiestan, sin distinción de colegio de origen, una visión extremadamente desvalorada de la carrera docente. La oposición entre vocación docente v/s pragmatismo laboral es recurrente. Los jóvenes reprochan la inexistencia de una verdadera vocación, planteando que los profesores pareciesen no estar ejerciendo la carrera que ellos teóricamente hubiesen querido ejercer: son en su mayoría, en el lenguaje de los jóvenes, «doctores o abogados fracasados».

Sin embargo, lo mismo que en el juicio hacia la calidad de la educación donde los propios jóvenes distinguían entre la «mala calidad de la educación en general» y «la buena educación que yo recibo», la distinción vuelve a aparecer al plantearse mayoritariamente que «mis profesores son buenos». En el caso de los estudiantes de colegios municipales se añade el hecho de que ellos tienen la visión de que es en este tipo de educación (la municipal) donde se manifiesta la verdadera vocación (quienes enseñan en el nivel municipal serían quienes tienen una honesta vocación por la función de enseñar).

Todos los jóvenes coinciden con el hecho de que las características que distinguen a un «buen profesor» son: a) conocer muy bien la materia que enseña (aspecto criticado habitualmente), b) saber transmitir fácilmente estos contenidos, y c) vincular éstos con la realidad (pertinencia). También se mencionan otros aspectos como ser motivador, cercano a los alumnos, con buen trato hacia ellos y desafiante frente al conocimiento para hacer pensar a los estudiantes.

Los estudiantes son concientes de que la profesión docente no es valorada socialmente, lo que hace que muchos jóvenes de buen desempeño y con capacidades para ejercer la docencia no quieran optar por este tipo de carrera. Específicamente se menciona la falta de incentivos que existen para optar a ser docente. Reconocen que la realidad chilena no incentiva seguir este tipo de profesión, y que ésta más bien está siendo ocupada por personas muchas veces sin vocación. Además son claros en mencionar que las cualidades requeridas en la actualidad para ser docentes son más exigentes que antaño, puesto que 
ellos deben ser capaces de atraerlos y motivarlos no sólo haciendo uso del conocimiento de la disciplina, sino también usando otro tipo de herramientas didácticas y tecnológicas. Deben adaptarse tanto a los nuevos conocimientos como a las características de los nuevos estudiantes.

\section{d) Apoyo al sistema escolar en la conformación de trayectorias educativas postsecundarias}

...el año pasado conocí a harta gente de las universidades por los movimientos. Entonces les pregunto a ellos por facebook, oye sabes que tengo tal problema, en Beauchef qué onda, como están los puntajes, cuestiones así (Hombre, colegio municipal).

Como ya lo hemos afirmado, todos los jóvenes quieren o sienten la obligación de entrar a la educación superior. Es este tipo de educación el que asegura, según lo declarado por ellos mismos, una mejor inserción laboral posterior. No hacerlo te discrimina. Incluso los estudiantes de colegios técnicos lo mencionan como la posibilidad para «poder surgir, para subir de nivel» y tener más estabilidad.

Todos los jóvenes plantean que el apoyo de sus colegios en el proceso de búsqueda de información y orientación vocacional es insuficiente. En el balance general, los jóvenes establecen que son ellos mismos los que buscan la información, afirmando que el colegio podría hacer más sin que quede plenamente claro en qué podría traducirse ese apoyo.

Un aspecto interesante, como también ya lo hemos referido anteriormente, es que los jóvenes provenientes de colegios municipales y subvencionados parecen apoyarse en "personas significantes» (hermanos mayores, primos, amigos, etcétera) que ya han pasado por el mismo proceso y que han mostrado éxito en sus «estrategias de búsqueda». El colegio aparece menos como un referente en este punto, a diferencia de lo que sucede con los estudiantes de colegios privados. Antes aparece incluso Internet y en el caso de los estudiantes que se han movilizado en el $2011,{ }^{4}$ la relación establecida con estudiantes universitarios durante las movilizaciones. Se menciona que los colegios hacen ferias y promueven información sobre eventos donde es posible encontrar información relevante para tomar decisiones. Los

4 Movilizaciones de estudiantes secundarios, preferentemente de colegios municipales, demandando una educación pública, gratuita y de calidad. 
estudiantes de estos colegios piden más apoyo aún a sus establecimientos, ya que entienden que la labor de los liceos es que sus estudiantes logren efectivamente ingresar a la educación superior.

Específicamente los estudiantes de colegios públicos mencionan que el tema del apoyo vocacional debiese ser más abierto, en tanto no todos quieren o pueden entrar a la universidad, ni todos piensan en carreras tradicionales. Mencionan que el apoyo que en general dan los colegios no se expresa en el caso de carreras menos tradicionales o más artísticas. Otro elemento más propio de este tipo de estudiantes se refiere a lo mencionado sobre las «personas significantes» como referente respecto a la toma de decisión sobre la educación superior. Este es el caso de los «hermanos mayores», quienes se convierten en el modelo a seguir (ellos ya hicieron el camino, ellos se esforzaron, triunfaron y saben lo que hay que hacer / es conveniente apoyarse en ellos).

Si bien en los colegios privados este proceso de búsqueda de información parece darse de manera más fluida y además aparecen distintos referentes proveedores de información, es importante mencionar lo relevado que aparece el colegio como instancia para ello. Un elemento interesante a este respecto es que si bien el colegio es referido como un gran proveedor de información para tomar decisiones, es criticado en cuanto él debiese preocuparse de apoyar en la identificación de las habilidades e intereses de los estudiantes, y no sólo informar sobre la variada oferta de carreras e instituciones de educación superior. La referencia programática de aplicar más tests vocacionales y de mejor calidad surge como una demanda. Este es el grupo de estudiantes con más conocimiento, lo que queda reflejado en que citan algunas páginas web donde ellos saben se encuentra la información sobre carreras.

Como se puede apreciar claramente, los estudiantes, independientemente de su nivel socioeconómico, tienen altas expectativas de continuar estudios superiores, y en este sentido imprimen en esta decisión una cuota alta de definición vocacional. El hacer primar - o intentarlo a lo menos - los gustos e intereses de los estudiantes como horizonte de lo que se quiere realizar laboralmente, es un elemento nuevo para algunos segmentos de la sociedad chilena, específicamente para los estudiantes de establecimientos más vulnerables.

Esta nueva configuración requiere de más apoyo, y así lo manifiestan los propios estudiantes, sobretodo si consideramos lo difícil que es elegir bien en un complejo mercado educativo como el chileno y más aun si consideramos que muchos de estos estudiantes no tienen 
una cercanía cultural con este tipo de educación, y si la tienen ésta es más bien reciente e inicial. Los estudiantes reconocen estar informados de la oferta educativa, pero generalmente restringida a instituciones y a carreras más tradicionales. Los estudiantes demandan una orientación más integral, donde no sólo se muestre la amplia gama de carreras, sino también las otras opciones que hoy existen de capacitación y perfeccionamiento. La orientación si bien se ha densificado, sigue estando casi exclusivamente circunscrita a dar información, y no a entregar las habilidades necesarias para diseñar e implementar proyectos de vida más complejos, situados en trayectorias más variadas y diversas que antaño, como aquellas de tipo «yo-yo» y ya no sólo tan lineales (Dávila, Ghiardo y Medrano, 2005; y Baeza, 2007) propios de sociedades postmodernas. Se demanda más apoyo también para aclarar los propios intereses y habilidades con el objetivo de tomar mejores decisiones a este respecto.

\section{e) Nuevas tecnologías}

...yo por ejemplo, se me echó a perder el computador y no podía estudiar porque siempre me manejo haciendo resúmenes en Word y es más fácil aprendérselo para mí escribiendo (Mujer, colegio privado).

Uso de internet. Las respuestas de los jóvenes no muestran elementos muy novedosos en esta materia. El uso de internet se da de manera muy generalizada para efectos de comunicación y realización de trabajos escolares. Respecto de la búsqueda de información ésta se da por interés propio o por demandas de naturaleza escolar. En materia escolar los jóvenes lo usan para entender cosas no clarificadas en clase o simplemente para hacer tareas. Respecto a cuestiones de información general, lo utilizan para bajar música o conocer más de sus artistas favoritos y leer noticias. Los programas más utilizados son, por lo tanto, aquellos que permiten la comunicación (Facebook, Skype, WhatsApp). El uso de Twitter es muy marginal, con una mayor prevalencia en los estudiantes de colegios privados. Messenger también disminuye su impacto; son los jóvenes de colegios subvencionados quienes señalan aún utilizarlo.

Relación nuevas tecnologías y colegio. Todos los jóvenes coinciden en torno a dos convicciones básicas: a) el uso de nuevas tecnologías es indispensable en el aula y en el proceso formativo global; y b) 
las nuevas tecnologías nunca podrán reemplazar al profesor presencial y al colegio en su dimensión física. Respecto al primer punto mencionan que las nuevas tecnologías les permiten enfrentar de mejor forma las exigencias que se les impone en el ámbito escolar; específicamente hacer tareas y presentaciones para el colegio. Respecto a Internet, el uso es más diverso, en tanto esta herramienta les permite ejercer distintas funciones que sirven de apoyo a las labores escolares. En primer lugar se menciona que la usan para buscar información que emerge como necesaria para lograr un buen desempeño escolar (para hacer tareas, para aclarar materias vistas en clases, etcétera). Además de ello, Internet es extendidamente usado para vincularse entre sí, lo que permite la realización de trabajos o definir grupos de interés que permita mantenerlos en contacto y dar aviso de situaciones escolares que los involucren (hay grupos de curso o de ramos electivos que los alumnos toman, etcétera). En este sentido este tipo de grupos mantienen el quehacer escolar más allá de los límites físicos y temporales que impone la escuela, permitiendo una comunicación fluida y práctica para afrontar mejor las exigencias escolares.

Acerca de la posibilidad de que las nuevas tecnologías puedan reemplazar en algún momento a la escuela como proveedor de conocimiento clave para la socialización cultural y la inserción social de las nuevas generaciones, los estudiantes manifiestan muy claramente que lo ven como imposible. Tanto el profesor como el colegio humanizan, aspecto que sería imposible si pensásemos sólo en el uso de nuevas tecnologías en el proceso de formación. La educación no es sólo conocimientos cognitivos, es una manera de socializar junto a otros, lo que hace indispensable la relación directa. A pesar de que hay jóvenes con más habilidades para auto formarse con el apoyo de herramientas como Internet, en general se requiere de una relación pedagógica, donde un experto acompañe y haga avanzar al estudiante, estando siempre disponible para aclarar sus dudas.

Los hallazgos del estudio nos señalan que las nuevas tecnologías y su uso cada vez más intensivo y masivo por las nuevas generaciones estarían resignificando el espacio escolar en algunas dimensiones relevantes. En primer lugar, aparece como una herramienta que es cada vez más imprescindible para lograr un buen desempeño académico. Tanto las TIC's como internet emergen como herramientas claves que estarían siendo internalizadas por el mundo escolar y consideradas como disponibles por sus estudiantes. Muchas exigencias escolares, 
sobre todo en población escolar de más recursos, suponen su accesibilidad cotidiana por parte de los estudiantes (en colegios privados los estudiantes mandan sus trabajos por e-mail al profesor hasta las 24:00 horas del día solicitado). Esto amplía el dominio escolar, colonizando tiempos tradicionalmente extraescolares.

También podemos observar que la conformación de grupos de estudiantes en las redes sociales con el objetivo de «sostener» información relevante respecto a los cursos está reforzando dicha tendencia. Al parecer, el mundo escolar es más amplio y difuso en sus límites que lo que lo era anteriormente (recordar como el cyberbulling mantiene el acoso fuera de los límites espaciales y temporales del horario escolar).

Otro aspecto que se podría atribuir al uso de las tecnología de la información y de la comunicación (TICS) y la racionalidad que tienen este tipo de herramientas tecnológicas (más interactivas, más autónomas en su uso, multimediales, etcétera) se puede observar en los referentes desde los cuales los estudiantes evalúan las disciplinas o ramos escolares y la actuación pedagógica de sus docentes. La demanda por clases más lúdicas, prácticas e interactivas puede verse reforzado por la racionalidad que imponen este tipo de herramientas y por el uso que los jóvenes de hoy le están dando (Pedró, 2006). Según este autor, las TICS estarían modificando la manera como las nuevas generaciones se están aproximando al conocimiento, por lo que ello podría concatenar a una demanda más exigente al colegio respecto a sus formas de entregarlo en su formato escolar.

Lo visto anteriormente no resta importancia a la necesidad de considerar el cómo estas nuevas tecnologías están modificando algunos aspectos de la educación actual y con ello imponiendo nuevas demandas que deben ser evaluadas por las políticas educativas con el objetivo de hacerlas más pertinentes y promotoras de una educación de calidad para todos.

\section{CONCLUSIONES: AGENDA DE INVESTIGACIÓN}

Los elementos que hemos descrito en este artículo, permiten descubrir, y en otros casos reforzar, una gama importante de inquietudes referidas a problemas de ajuste no resueltos entre los jóvenes y el sistema educativo. Estos eventuales «desajustes» pueden formar globalmente una «agenda» de preocupaciones que se traduzca en investigaciones avanzadas susceptibles de ser impulsadas en el plano académico. Ellas también debiesen interesar a quienes desde el diseño de las 
políticas públicas se encuentran precisamente examinando la pertinencia del modelo y estrategias de nuestro actual sistema educacional.

Atendiendo a este espíritu, consideramos relevante precisar ciertos temas que dada la naturaleza y restricciones del estudio no se logró profundizar, pero que dada su importancia y presencia a nivel de los resultados del trabajo, ameritan ser parte de una agenda futura de investigación.

En este contexto destacamos los siguientes fenómenos.

a) Desajustes entre promesa de movilidad social e integración efectiva de los jóvenes. En materia de movilidad social es indispensable indagar sobre las consecuencias simbólicas de las promesas de la movilidad social asociadas a la educación (v.g. equilibrio entre expectativas de inserción en el mercado laboral y participación laboral efectiva). En esta línea, debiesen estudiarse eventuales mecanismos de regulación al mercado del trabajo con la finalidad de que los avances en educación tengan un correlato en el sistema, haciendo valer efectivamente la lógica de la meritocracia. En materia educativa es urgente una reflexión sobre las expectativas desmedidas depositadas en lo educativo, preguntándose si, por ejemplo, el sistema de aseguramiento de la calidad sabrá abordar adecuadamente los problemas estructurales de la desigualdad de resultados y segregación. Desde una perspectiva más sociológica, es adecuado pensar en las consecuencias de un modelo que ofrece simbólicamente altas expectativas de integración a través de la educación preferentemente universitaria, pero que carece de mecanismos para garantizar que esta integración opere con certitud. Planteado de otra forma, es preciso preguntarse por el comportamiento de los «optimistas de hoy» si es que sus expectativas de movilidad no son satisfechas a futuro.

b) Desajustes entre la promesa de futuro versus el «eterno presente» del imaginario juvenil. El sistema educativo y experiencia juvenil parecen transitar en dimensiones temporales de naturaleza y características extremadamente antagónicas. Por un lado, la educación se ordena en torno a una visión temporal estructurada sobre la base de un futuro no bien precisado, que es rigor una «promesa de futuro». Por el contrario, los jóvenes, a pesar de ser sensibles a la idea de un proyecto que se construye en el tiempo, se sienten más interpelados por una inmediatez que les resulta mucho más inteligible. De esta forma, en el imaginario juvenil, «lo valioso y lo útil parecer ser lo vivible y lo 
verificable hoy». Por tal motivo, lo anterior exige pensar formas de conciliación de ambas perspectivas que permitan, en el caso de la educación, responder a exigencias juveniles propias del hoy, y, en el caso de los propios jóvenes, reinstalarlos simbólicamente en una concepción de tiempo extenso o extendido. De alguna forma la incorporación de las tics en el mundo cotidiano de los jóvenes y progresivamente en el espacio escolar está explicitando esta tensión. La dimensión temporal y las representaciones de él pasan a ser un tema que debe ser considerado en los estudios de juventud y del mundo educativo.

c) Desajustes entre civismo en abstracto y participación efectiva. A pesar del fuerte consenso con la idea de que la proximidad con las instituciones democráticas, la disposición a participar en los procesos políticos y el desarrollo de lo que normalmente se denominan las virtudes públicas dependen en gran medida de la educación que los jóvenes reciben en el colegio - puesto que es en el colegio donde se vive concretamente la alteridad y se adquieren los útiles fundamentales para la vida en comunidad - el sistema educativo sigue reforzando una estrategia de neutralidad que impide que los jóvenes puedan desarrollar un verdadero sentimiento de solidaridad colectiva. Esta neutralidad paradojalmente también se apoya en una concepción cívica esencialista y abstracta que pareciera exigir a los jóvenes un comportamiento natural que no requiere ni de un aprendizaje ni de una práctica. Lo anterior pone en evidencia la necesidad de reorientar el rol del colegio en relación a la formación para la ciudadanía, desarrollando líneas educativas más claras y más pertinentes que se estructuren a partir de desafíos precisos en los ámbitos de alteridad, asociatividad, titularidad de derechos y responsabilidades que de ello se deriven; entre otros aspectos.

En este marco no es menos relevante plantearse el qué hacer, desde el sistema educativo, respecto del voto como mecanismo base de la democracia representativa y también el cómo reforzar la cohesión social, la prosocialidad y la empatía en contrapunto al individualismo cultural que hemos descrito en la primera sección de este artículo.

SANTIAGO (CHILE), NOVIEMBRE 2012

RECIBIDO: DICIEMBRE 2012

ACEPTADO: FEBRERO 2013 


\section{REFERENCIAS BIBLIOGRÁFICAS}

BAEZA, JORGE (2007): «La construcción de trayectorias en sociedades menos reguladas: desafíos al trabajo de orientación escolar en educación secundaria». Foro Educacional No12. Santiago: Universidad Católica Silva Henríquez.

BAJOIT, GUY (2003): Le changement social: approche sociologique des sociétés occidentales contemporaine. Paris: Armand Colin.

Castillo, Jorge y Gustavo Cabezas (2010): «Caracterización de jóvenes primera generación en educación superior». Calidad en la Educación N³2. Santiago: Consejo Nacional de Calidad de la Educación.

DÁVILA, ÓsCAR, FELIPE GHIARDO y CARLOS MEDRANO (2005): Los desheredados: trayectorias de vida y nuevas condiciones juveniles. Valparaíso: Ediciones CIDPA.

DUBET, FRANÇOIS (2005): La escuela de las oportunidades ¿Qué es una escuela justa? Barcelona: Gedisa Editorial.

Duru-Bellat, MARIE (2009): Le mérite contre la justice. Paris: Presses de la Fondation Nationale des Sciences Politiques.

PEDRÓ, FrANCESC (2006): «Aprender en el nuevo milenio. Un desafío a nuestra visión de las tecnologías y la enseñanza». OCDE-CERI.

Guell, PEDRO (2008): «Comentarios V encuesta nacional de juventud». Revista de Desarrollo Humano No41. Santiago: PNUD.

InSTITUTO NACIONAL DE LA JUVENTUD (2011): Imaginarios juveniles de la política. Santiago: INJUV.

- (2010): VI encuesta nacional de juventud. Santiago: INJUV.

LECHNER, NORBERT (2004): «Cultura juvenil y desarrollo humano». Revista de Estudios sobre Juventud $\mathrm{N}^{\circ}$ 20. México: IMJ.

MONTES, NANCY y MARÍA ALEJANDRA SENDÓN (2006): «Trayectorias educativas de estudiantes del nivel medio. Argentina a comienzos del siglo XXI». Revista Mexicana de Investigación Educativa, Vol. 11, N²9. México: Consejo Mexicano de Investigación Educativa.

Nussbaum, Martha (2010): Sin fines de lucro. Por qué la democracia necesita de las humanidades. Buenos Aires: Katz Editores.

TeZanos, José FéliX, JuAn José Villalón y AinOA QuiÑones (2011): Estudios Delphi sobre tendencias económicas, sociales y políticas. Madrid: Editorial Sistema.

THEZÁ, MARCEL y ALEJANDRO REINOSO (2005): «Jóvenes y violencia: notas para su comprensión y análisis». Persona y Sociedad, Vol. XIX, №1. Santiago: Universidad Alberto Hurtado. 